\title{
School Refusal Behavior in Young Children
}

\author{
Christopher A. Kearney \\ Gillian Chapman \\ L. Caitlin Cook
}

\begin{abstract}
School refusal behavior is defined as any child-motivated refusal to attend classes and/or difficulty remaining in classes for an entire day. Although many researchers have focused on older children and adolescents in their samples, few have specifically focused on young children aged 5-9 years (i.e., kindergarten to third grade). In this article, a general description is made of school refusal behavior, and illustrative data from 55 young children with school refusal behavior are provided. Recommendations for assessing and treating this young population are made as well.

Keywords: School refusal behavior, young children.
\end{abstract}

School refusal behavior refers to a child-motivated refusal to attend school and/or difficulty remaining in classes for an entire day. School refusal behavior is a particularly devastating condition for many families because the behavior often leads to increased conflict, academic and legal problems, friction with school officials, and lost time from work. The problem often involves a myriad of internalizing and externalizing behaviors, making conceptualizations based on the form of behavior traditionally difficult. In addition, such heterogeneity of behavior has stifled the development of consensus opinions regarding the assessment and treatment of this population. For a more detailed description of this population, the reader is referred to other sources (Kearney, 2001; 2003; 2005; Kearney \& Albano, 2000; Kearney \& Silverman, 1996).

Many of the assessment and treatment studies available to clinicians regarding youths with school refusal behavior include samples of older children and adolescents. This is often done so that information can be collected regarding cognitive distortions, mood changes, intricate family dynamics, and other variables that are more difficult to assess in younger children. As a result, less information is available regarding the youngest section of youths with school refusal behavior. In particular, less information is available about the specific diagnostic and functional characteristics of children aged 5-9 years who refuse school.

In our outpatient clinic for youths with school refusal behavior, we have examined children and adolescents of various ages using structured diagnostic interviews, child selfreport measures, parent and teacher checklists, daily logbooks, and consultations with school officials. In doing so, we have accumulated data on 55 youths with school refusal behavior who are aged 5-9 years. In this paper, we summarize some of the diagnostic, functional, and parent data to provide researchers and clinicians with a snapshot of this population. In addition, assessment and treatment recommendations are made for this younger group.

\section{$\underline{\text { Diagnostic Data }}$}

Diagnoses for this group were made by using child and parent versions of the Anxiety Disorders Interview Schedule for DSM-IV (Silverman \& Albano, 1996). This interview 
provides diagnostic information for various internalizing and externalizing behavior problems, with a particular emphasis on anxiety disorders. The interview has demonstrated excellent reliability and validity across different psychometric studies (DiBartolo, Albano, Barlow, \& Heimberg, 1998; Silverman, Saavedra, \& Pina, 2001; Wood, Piacentini, Bergman, McCracken, \& Barrios, 2002). Information from the child and parent versions is integrated to provide a comprehensive diagnostic picture.

Regarding youths from our clinic who are aged 5-9 years and who have school refusal behavior, primary combined diagnoses include:

- Separation anxiety disorder (53.7\%)

- No diagnosis $(22.2 \%)$

- Generalized anxiety disorder (9.3\%)

- Specific phobia $(9.3 \%)$

- Social phobia/avoidant disorder (3.7\%)

- Enuresis (1.9\%)

The most common secondary diagnosis was generalized anxiety disorder, which affected $20.4 \%$ of youths. Other secondary diagnoses were scattered but largely included oppositional defiant disorder (13.0\%) and separation anxiety disorder (9.3\%). Other disorders sometimes thought to be common in this population, including attention deficit hyperactivity disorder and learning disorders, were not prevalent.

\section{$\underline{\text { Functional Data }}$}

Although assessing the forms of school refusal behavior is useful, Kearney and colleagues have devised methods to also assess the function of this behavior in youths. The reader is referred to other sources for a full description of our functional model and methods of assessment (Kearney, 2001; Kearney \& Albano, 2000; Kearney, Lemos, \& Silverman, 2004). Briefly, however, the functional model of school refusal behavior stipulates that such behavior is generally maintained by one or more of the following conditions:

- $\quad$ To avoid school-based stimuli that provoke a general sense of negative affectivity (anxiety and depression)

- $\quad$ To escape aversive school-based social and/or evaluative situations

- To pursue attention from significant others

- $\quad$ To pursue tangible reinforcers outside of school

Although functional assessment of this population generally involves a combination of measures, a quick, descriptive method for determining the primary reason a particular child is missing school is the School Refusal Assessment Scale, now revised (Kearney, 2002; Kearney $\&$ Silverman, 1993). Child and parent versions of the scale are available, and item means from each version are combined to derive an overall functional profile. Studies have indicated the scales to have good test-retest and interrater reliability as well as concurrent and construct validity. 
Regarding youths from our clinic who are aged 5-9 years and who have school refusal behavior, we have found that the following primary functions apply:

- $\quad$ Pursuit of attention from significant others (55.1\%)

- $\quad$ Avoidance of stimuli that provoke negative affectivity (20.4\%)

- $\quad$ Pursuit of tangible reinforcers outside of school $(20.4 \%)$

- $\quad$ Two or more functions of equal strength (4.1\%)

- $\quad$ Escape from aversive social and/or evaluative situations $(0.0 \%)$

In general, younger children often refuse school to be with parents or other significant attachment figures at home. This may result from recent and critical family transitions such as parental illness or move, but may also result from extended parental acquiescence to noncompliant behavior. Indeed, in a previous diagnostic study, oppositional defiant disorder was found to be most associated with this functional condition. However, the school refusal behavior of youths aged 5-9 years is also marked by desires for negative reinforcement as well as desires to pursue tangible reinforcers outside of school. Interestingly, none of the children in our sample refused school to escape aversive social and/or evaluative situations. This may be due to the fact that social anxiety is a largely developmental phenomenon that begins to more fully emerge in later elementary school years (Kearney, 2005).

\section{$\underline{\text { Parent Data }}$}

Parent data for this group included scores from measures of child internalizing and externalizing behavior problems as well as family dynamics. Measures included the Child Behavior Checklist (CBCL) (Achenbach \& Edelbrock, 1991) and Family Environment Scale (FES) (Moos \& Moos, 1986), the latter of which assesses for ten dimensions of family functioning: cohesion, expressiveness, conflict, independence, achievement orientation, intellectual-cultural orientation, active-recreational orientation, moral-religious emphasis, organization, and control. Each measure has demonstrated excellent psychometric properties.

On the CBCL, T scores of internalizing and externalizing behavior problems are derived. T scores of 67-70 are considered to be in the "borderline clinical range" and T scores of 70+ are considered to be in the "clinical range." For our sample of younger children with school refusal behavior, mean T scores for the internalizing and externalizing scales were 69.7 and 58.4, respectively. In fact, $50.9 \%$ of these youths were rated in the clinical range for internalizing behavior, compared to only $11.3 \%$ of youths in the clinical range for externalizing behavior.

On the FES, T scores of 50 are considered to be average functioning. In our sample of young children with school refusal behavior, FES T scores generally surrounded the norm, but two dimensions of family functioning were at least one-half of a standard deviation from the norm. In particular, independence T scores (40.6) were rated much lower than the norm, whereas moral-religious emphasis $\mathrm{T}$ scores (57.0) were rated much higher than the norm. 


\section{$\underline{\text { Implications for Assessment }}$}

Young children with school refusal behavior appear to be characterized largely by separation anxiety, attention-seeking motives, and oppositional behavior. Often these characteristics are manifested by willful, stubborn, manipulative behaviors designed to force parental acquiescence to demands of various sorts. The fact that many of these families are marked by low levels of independence, or high levels of dependence, serves to reinforce this characterization. A common scenario is a child who refuses to attend school as well as other activities such as sleepovers, and who demonstrates oppositional behavior such as temper tantrums to accomplish these goals. Families with low independence, or high enmeshment, may be more predisposed toward reinforcing this scenario compared to other family types.

As such, clinicians are encouraged to assess for a broad spectrum of attention-seeking behaviors in young children with school refusal behavior. This may be accomplished by use of interviews, parent and teacher data, and especially direct observations. Although direct observations in natural settings can be time-consuming, observations of in-session behaviors may be quite instructive. In particular, clinicians may wish to watch for behavior that is clingy, noncompliant, defiant, demanding, and manipulative in nature. In addition, many young children of this type will refuse to be assessed without the company of a parent, and many will refuse to answer questions even with a parent present. In our experience, much of this behavior is designed to force parental acquiescence to demands to leave the clinic setting and to maintain the status quo within the family, which clearly benefits the child.

Although attention-seeking behavior predominates among young children, our data indicate that other behavior sets and functions characterize significant subgroups. Many young children with school refusal behavior, for example, especially those new to a school setting, will demonstrate anxiety-based behaviors designed to avoid aversive school-based stimuli. Although these stimuli are often difficult to pinpoint, these young children often have concerns about transitions between school settings (e.g., classroom and cafeteria) as well as concerns about riding a school bus or eating with large groups of people. These children will often cry, show somatic complaints such as headaches and stomachaches, and persistently ask to be relieved of school attendance. Some of these children will attend school but do so with great dread. Assessment of this subgroup should also focus on information from multiple sources, but direct observations of anxiety-based behavior in the clinic setting would be highly informative as well.

Another group of youngsters who miss school do so for tangible reinforcers outside of school. These children are not generally anxious, nor do they particularly wish to be with parents outside of school. Instead, these children are drawn more to stimuli such as playing with friends, riding a bicycle, sleeping late, watching television, or participating in other activities during a school day. Specific diagnoses and behavior patterns are less identifiable in this group of young children, but clinicians should be especially watchful of behaviors designed to accrue substantial incentives from parents and others for prosocial behaviors such as attending school or going to bed on time. 


\section{$\underline{\text { Implications For Treatment }}$}

Treatment of youths who refuse school necessarily requires a layered and prescriptive approach, preferably one that is based on the function of behavior (for details, see Kearney, 2001; Kearney \& Albano, 2000). For youngsters who predominately show attention-seeking behavior and its concomitants, we recommend a parent-based treatment approach that focuses heavily on modification of parent commands and establishment of specific routines in the morning and, if necessary, during the day. We also advocate the use of incentives and disincentives for school attendance and nonattendance, and, in unusual cases, forced school attendance.

The purpose of these treatment techniques is to help parents develop skills necessary to confront and modify a child's manipulative and oppositional behavior. In addition, treatment is designed to increase parental resolve and to educate parents about the shortcomings of previous strategies of avoidance and acquiescence. In some cases, work with parents involves a modification of their own behaviors, which may include elements of separation anxiety, depression and withdrawal from parenting, and marital conflict leading to poor child supervision and inconsistency of consequence administration. In other cases, clinicians will need to be sensitive to cultural issues that predispose some families toward greater dependence and possible acquiescence to child demands.

For youths who are anxious about school, treatment can be more child-based and focus on somatic management techniques (e.g., relaxation training and breathing retraining), education about anxiety and its components, cognitive intervention for youths who have sufficient skills to respond to this type of therapy, and exposure-based practices. Exposurebased practices typically involve gradual reintroduction into regular classroom settings, which may occur for increasing amounts of time or in greater physical proximity to a child's classroom (e.g., starting attendance in the school library before gradually transitioning to the classroom).

For youths who refuse school for tangible reinforcers outside of school, treatment may be more family-based in nature. This type of treatment includes contingency contracting to increase incentives for school attendance and to decrease incentives for school nonattendance, increased supervision of a child, communication skills training to reduce conflicts at home, and peer refusal skills training so that youngsters know how to effectively address offers from peers to miss school.

\section{$\underline{\text { Final Comments }}$}

Assessing and treating youths with school refusal behavior are traditionally difficult clinical tasks. When a child with school refusal behavior is young, however, these tasks are made even more difficult by his or her limited cognitive development, inability to express or identify emotions, and willingness to display overt attention-seeking misbehaviors without embarrassment. A thorough assessment approach that identifies both the form and function of school refusal behavior is thus recommended, as is a prescriptive and innovative treatment 
approach that is flexible enough to address the many layers of problems that a clinician will likely encounter in this population.

\section{References}

Achenbach, T.M., \& Edelbrock, C.S. (1991). Manual for the Child Behavior Checklist and Revised Child Behavior Profile. Burlington, VT: University of Vermont.

DiBartolo, P.M., Albano, A.M., Barlow, D.H., \& Heimberg, R.G. (1998). Cross-informant agreement in the assessment of social phobia in youth. Journal of Abnormal Child Psychology, 26, 213-220.

Kearney, C.A. (2001). School refusal behavior in youth: A functional approach to assessment and treatment. Washington, DC: American Psychological Association.

Kearney, C.A. (2002). Identifying the function of school refusal behavior: A revision of the School Refusal Assessment Scale. Journal of Psychopathology and Behavioral Assessment, 24, 235-245.

Kearney, C.A. (2003). Bridging the gap among professionals who address youth with school absenteeism: Overview and suggestions for consensus. Professional Psychology: Research and Practice, 34, 57-65.

Kearney, C.A. (2005). Social anxiety and social phobia in youth: Characteristics, assessment, and psychological treatment. New York: Springer.

Kearney, C.A., \& Albano, A.M. (2000). When children refuse school: A cognitive-behavioral therapy approach/Therapist's guide. San Antonio, TX: The Psychological Corporation.

Kearney, C.A., Lemos, A., \& Silverman, J. (2004). The functional assessment of school refusal behavior. The Behavior Analyst Today, 5, 275-283.

Kearney, C.A., \& Silverman, W.K. (1993). Measuring the function of school refusal behavior: The School Refusal Assessment Scale. Journal of Clinical Child Psychology, 22, 85-96.

Kearney, C.A., \& Silverman, W.K. (1996). The evolution and reconciliation of taxonomic strategies for school refusal behavior. Clinical Psychology: Science and Practice, 3, 339354.

Moos, R.H., \& Moos, B.S. (1986). Family Environment Scale manual (2nd ed.). Palo Alto, CA: Consulting Psychologists Press.

Silverman, W.K., Saavedra, L.M., \& Pina, A.A. (2001). Test-retest reliability of anxiety symptoms and diagnoses with the Anxiety Disorders Interview Schedule for DSM-IV: Child and Parent Versions. Journal of the American Academy of Child and Adolescent Psychiatry, 40, 937-944. 
Wood, J.J., Piacentini, J.C., Bergman, L., McCracken, J., \& Barrios, V. (2002). Concurrent validity of the anxiety disorders section of the Anxiety Disorders Interview Schedule for DSM-IV: Child and Parent Versions. Journal of Clinical Child and Adolescent Psychology, 31, 335-342.

All authors may be reached at:

Department of Psychology

University of Nevada, Las Vegas

4505 Maryland Parkway

Las Vegas, NV 89154-5030

Telephone: 702-895-3305

Fax: 702-895-0195

Email addresses for each author are as follows:

Kearney: ckearney@ccmail.nevada.edu

Chapman: chapmang@unlv.nevada.edu

Cook: cook16@unlv.nevada.edu

Advertising in the International Journal of Behavior \& Consultation Therapy

The prices for advertising in one issue are as follows:

1/4 Page: $\$ 50.00 \quad 1 / 2$ Page: $\$ 100.00 \quad$ Full Page: $\$ 200.00$

If you wish to run the same ad in multiple issues for the year, you are eligible for the following discount:

1/4 Pg.: \$40 - per issue

1/2 Pg.: \$75 - per issue

Full Page: \$150.00-per issue

An additional one time layout/composition fee of $\$ 25.00$ is applicable

For more information, or place an ad, contact Halina Dziewolska by phone at (215) 462-6737or e-mail at: halinadz@hotmail.com 